\title{
Corynebacterium amycolatum
}

National Cancer Institute

\section{Source}

National Cancer Institute. Corynebacterium amycolatum. NCI Thesaurus. Code C86308.

A species of aerobic or facultatively anaerobic, Gram-positive, coryneform, pleomorphic bacilli assigned to the phylum Actinobacteria. This species is nonmotile, non-spore forming, catalase and urease positive, oxidase negative, non-acid fast, non-lipophilic, and does not produce hippurate. C. amycolatum is commensal to the human skin and mucous membranes and is known to cause prosthetic joint related infections, and pneumonia, especially in immunocompromised persons. 